\title{
An Improved Greedy Parameter Stateless Routing in Vehicular Ad Hoc Network
}

\author{
Kavita, Neera Batra, Rishi Pal Singh \\ CSE Department, MMU Mullana, Ambala, India
}

\section{Article Info \\ Article history: \\ Received May 26, 2017 \\ Revised Dec 20, 2017 \\ Accepted Feb 26, 2018}

Keyword:

AODV

GPSR

Improved GPSR

VANET

\begin{abstract}
Congestion problem and packet delivery related issues in the vehicular ad hoc network environment is a widely researched problem in recent years. Many network designers utilize various algorithms for the design of ad hoc networks and compare their results with the existing approaches. The design of efficient network protocol is a major challenge in vehicular ad hoc network which utilizes the value of GPS and other parameters associated with the vehicles. In this paper GPSR protocol is improved and compared with the existing GPSR protocol and AODV protocol on the basis of various performance parameters like throughput of the network, delay and packet delivery ratio. The results also validate the performance of the proposed approach.
\end{abstract}

Copyright (C) 2018 Institute of Advanced Engineering and Science. All rights reserved.

\section{Corresponding Author:}

Kavita,

Department of Computer Science and Engineering,

Maharishi Markandeshwar University,

Mullana, Ambala, India.

Email: kavi4009@gmail.com

\section{INTRODUCTION}

VANET (Vehicular Ad hoc networks) is a type of Mobile Ad hoc Network (MANET). Each node acting as router to transfer information from one node to another node. In the VANET network, movement of node is based on the geographical area [1].VANET has given birth to many attractive applications. "Collision Avoidance” is one of its applications. Most of the road accidents are resulting from vehicles parting the road or traveling rashly through intersections. Inter vehicular communications and infrastructure to vehicular communication as discussed above can save many road accidents and therefore can save many human lives. The worst traffic accident occurs when a number of vehicles strike each other after a single accident suddenly halts traffic. In collision avoidance technique whenever a single vehicle lower its speed because of any reason, it broadcasts its position and other related information to all other vehicles. Furthermore, long waiting hours in traffic increase time wastage for the drivers. An important decline in numbers may be attained during VANET [5]. In this approach vehicles collect the desired information about the current traffic from surrounding environment and send it over network. Using this uploaded information traffic agencies helps in controlling congestion. In this approach, each vehicle calculates the number of its neighbor vehicles and their averages speeds and then sends this information to other vehicles in order to prevent them approaching the busy location. Moreover in some cases, the message may be communicated by those vehicles which are moving in anotherpath thus it can be communicated earlier to the anyautomobile mediumin the direction of the overcrowdingsite. Information like climate, road surface, manufacture zones, railways lines, and emergency vehicle signal is also collected by vehicles.

Greedy Perimeter Stateless Routing (GPSR) is a routing protocol that depends on the geologicallocation of nodes which is also required for vehicular ad-hoc network (VANET) [2]. GPRS attains neighbor vehicle message by using GPS positioning apparatus instead of obtaining huge routing information 
to maintain the message in routing table. In this routing protocol, every node transmits its location information periodically to the neighboring nodes. The information received by neighboring nodes is stored in the form of tables stored at those nodes. In order to promote the desired packet effectively, GPSR utilizes the information of nearest neighbor of destination [3]. Every node in GPSR has information of its location and his neighbors. The information about location of node provides helps to obtain better routing. All the neighboring nodes facilitate in making the forwarding decision in suitable way without snooping with the information related to topology. The benefit of GPSR is that it keeps the currently existing location of the forwarding node. This can helps to send the packet in shot time interval and also reduce the distance among destination nodes. Moreover, there are some demerits as well. In this GPSR protocol, few topologies results in decreasing the packet from moving to specific range from the destination [4]. Also, this protocol will not work if there will no nearest neighbor present to destination. GPSR is not appropriate for those ad-hoc networks where nodes are moving highly and the node will be unable to maintain its one hop neighbor's information as the other node can move out of its coverage area or range because of the higher mobility of dynamic nodes. It can result in loss of data packets.

\section{LITERATURE REVIEW}

In [1] geo AODV routing protocol has been proposed. This proposed protocol is similar with location aided routing which deploys GPS coordinates to reduce the area of search which is utilized through the meansof discovery process. GeoAODV is used to dynamically distribute the position data between the nodes in the network. Also, in this work, performance of proposed protocol is compared with other LAR, AODV routing protocol. In [2] location aided protocol has been proposed. The proposed approach is utilizing the position information in order to enhance the routing protocol performance for any kind of ad-hoc network. The proposed protocol reduces the area of search by utilizing the position data. The proposed protocol moves in the direction where the final node is supposed to be located. Also, location aided routing modifies the process of finding the route, so that only those nodes will rebroadcast the request message that belongs to the search area. Due to this, routing message are reduces.

Shen et al. [3] proposed Location-based Efficient Routing protocol has been presented. The proposed protocol divides the network field into separate zones and arbitrarily selects the node which may act as intermediate relay nodes and these nodes makes non traceable anonymous route. Furthermore, it conceal the data receiver between several receivers to make stronger destination and source ambiguitysafety. In [4], a novel routing scheme depending on GPSR routing protocol has been presented. The simulation has been executed by using VANET MobiSim. The comparison has been presented among the proposed protocol and AODV on network simulator NS2. The result demonstrates that the proposed GPSR routing scheme performs better in terms of end to end delay andpacket delivery ratio. OLSR and DSDV protocols have been presented in [5]. For simulation purposes, network simulator 3 and 802.11p standard and Two Ray Ground Propagation Loss Model are used. The performance is compared by already existing protocol and result demonstrated that the proposed OLSR protocol is better. In this analysis [6], it involves the two types of MAC /PHY specifications that isIEEE 802.11g and the IEEE 802.11a. In the first experimental design, signal strength has been calculated which is produced by analyzed devices. The second design illustrates the quality of service of V2V communications of these devices.

The study in [7] analyzes the delay in the delivery the information only for the purpose of roadside unit (RSU) deployment in VANET network. Also in this paper, a model has been designed which helps to explain the relation among delay and deployment distancebetween roadside neighbor units. Furthermore, the designed model considers the speed of vehicle, its density and some other parameters. The correctness and accuracy of the proposed model is confirmed and the effectsseveral parameters on the average delay are investigated through simulation results.

In [8], new scheme has been presented which is depends on the prediction of velocity and selective forwarding. The sender choices the best candidate which will rebroadcast the message to other vehicle. In this technique, low overhead has been generated. This work proposed a broadcasting algorithm for VANETs. The proposed algorithm is depending on the kalman filtering. The simulations results indicate that the proposed technique may improve the delivery ratio and decrease the end to end delay

\section{PROPOSED METHODOLOGY}

In the proposed approach the link between source and destination is computed considering various parameters. These parameters are acceleration of the target vehicle, velocity of source and target vehicle, distance between the source and the target vehicle and the direction of the target vehicle. For the calculation 
of the path, firstly a virtual line among the destination and source is considered using the two point line equation.

$$
y-y_{1}=\left[\left(y_{2}-y_{1}\right) /\left(x_{2}-x_{1}\right)\right]\left(x-x_{1}\right)
$$

Now, the distance of each neighbor of the source node is calculated from the destination node using equation 2 and compared the distance with the source to destination distance. Nodes satisfying the following criterion will only be considered for the path selection process:

1. Velocity of the source node is less than destination node

2. Acceleration of destination node is more than source node

3. Destination or target node lies within the range of source node

4. Direction of both source node and the target node are same

After the initial selection of nodes, the perpendicular distance of the node with the virtual line is calculated using equation 3 and the node with the least distance is selected for route discovery and the source node is updated with the selected node and process continues until destination is reached. This is because the angle among the destination and sourcenode is minimized in order to make the GPSR protocol to be angle aware.

$$
\begin{aligned}
& \text { dist }=\sqrt{ }\left(\left(x_{2}-x_{1}\right)^{2}+\left(y_{2}-y_{1}\right)^{2}\right) \\
& \text { perpendicular distance }=\frac{|A m+B n+C|}{\sqrt{\left(A^{2}+B^{2}\right)}} \\
& \text { from a line } A x+B y+C=0 \\
& \text { and } m \text { and } n \text { are the } x \text { and } y \text { coordinates of concern node }
\end{aligned}
$$

Figure 1 show the path selection procedure based on the perpendicular distance of the nodes from the virtual line.

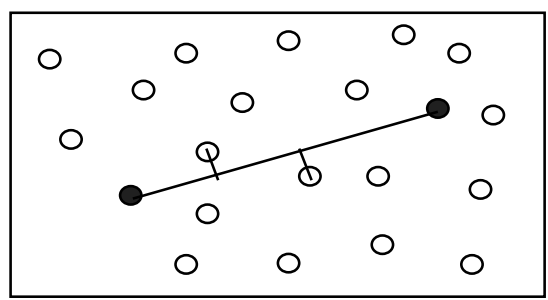

Figure 1. Path Calculation

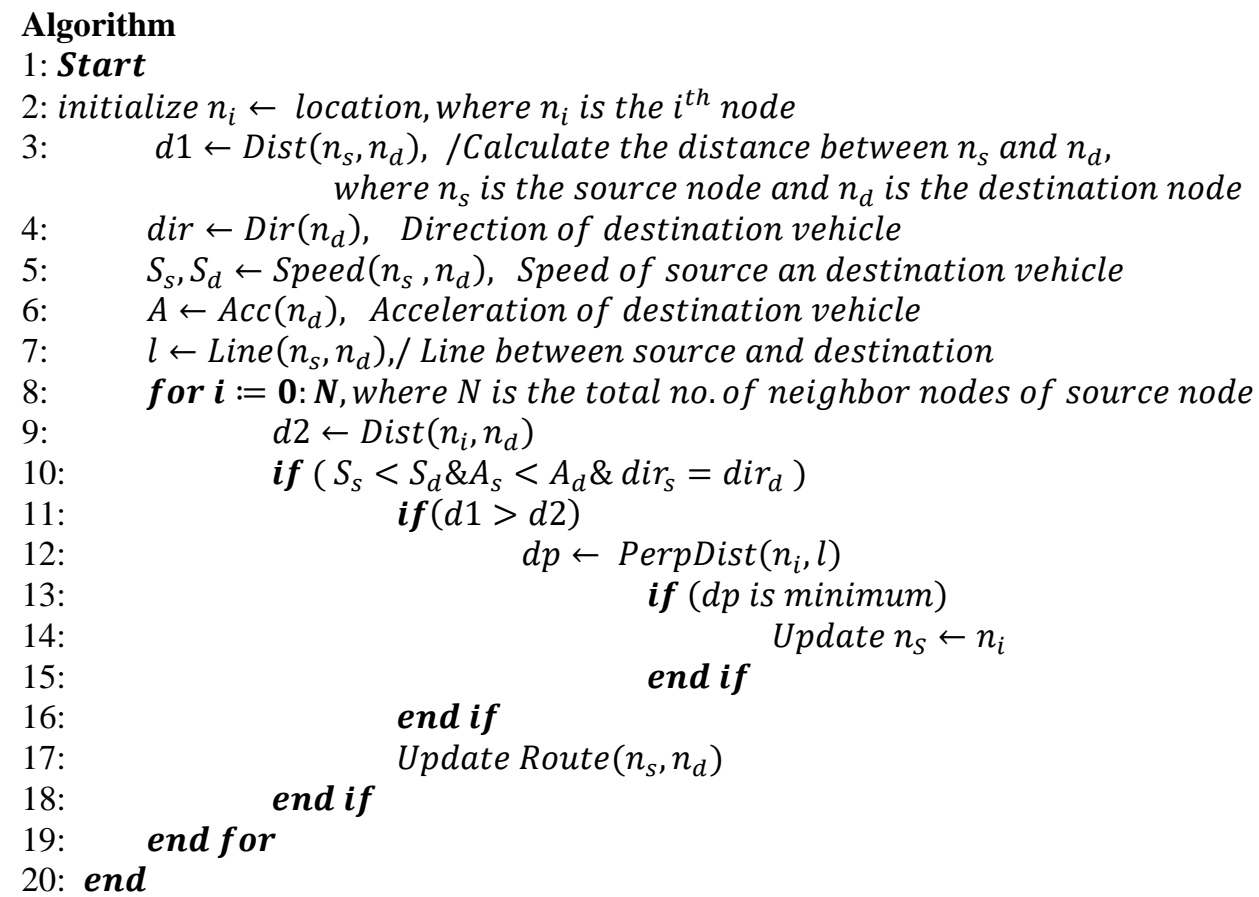




\section{SIMULATION ENVIRONMENT}

Table 1 shows the parameters set up for creating the simulation environment.

Table 1: Simulation Parameters

\begin{tabular}{cc}
\hline Channel & Wireless Channel \\
\hline Propagation Model & Two Ray Ground \\
Mac & IEEE 802.11 \\
Antenna & Omni Directional Antenna \\
Number of Vehicles & $10 \sim 50$ \\
Simulation Time & $50 \mathrm{~s}$ \\
\hline
\end{tabular}

\section{RESULTS AND DISCUSSIONS}

The proposed methodology is implemented using the network simulator ns2.35. The Vehicular Ad hoc Network environment is created and performance of GPSR and Improved GPSR is compared with the AODV protocol on parameters like throughput of the network, packet delivery ratio and end to end delay. Figure 2 to 4 shows the graphs of comparison between these protocols on the basis of various performance parameters varies accordingto the number of nodes.

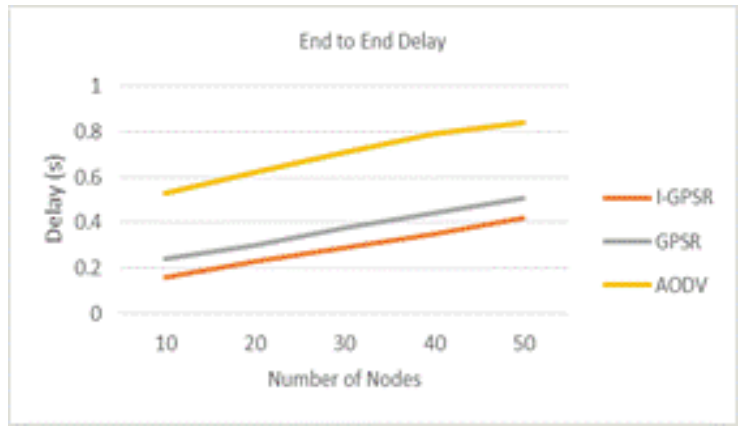

Figure 2. End to End Delay

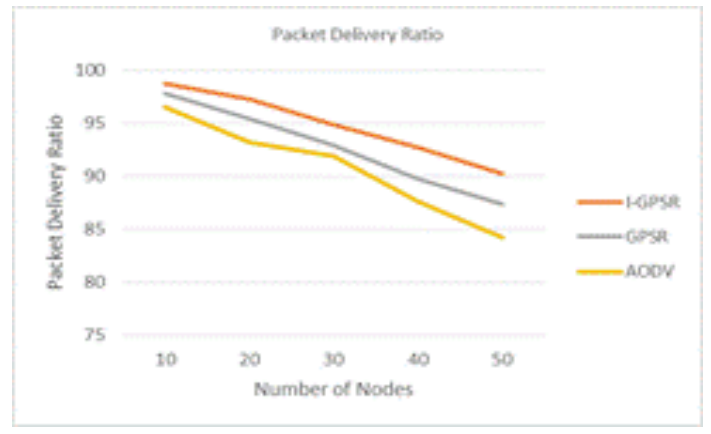

Figure 3. Packet Delivery Ratio

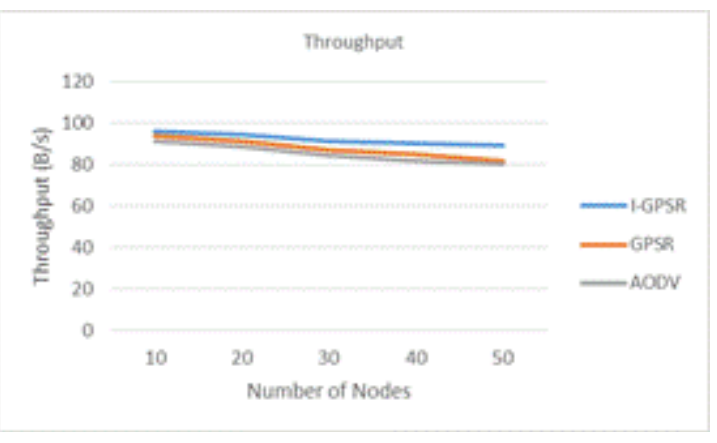

Figure 4. Throughput of the Network

\section{CONCLUSION}

The paper tries to present a comparative analysis of existing AODV and GPSR protocol and improved GPSR protocol in terms of their performance. A VANET environment was created and vehicular movement were simulated. The above discussed protocols were used for communication and the performance of the system was analyzed. For a similar environment, it was found that the improved GPSR protocol outperforms the other two protocols in terms of throughput, network delay and packet delivery ratio. This encourages us to further explore the proposed improved GPSR protocol in other environment. In future, hybrid protocols can be developed by exploiting the better features of improved GPSR and the traditional algorithms. 


\section{REFERENCES}

[1] Hnatyshin, Vasil, Malik Ahmed, Remo Cocco, and Dan Urbano. "A comparative study of location aided routing protocols for MANET." In Wireless Days (WD), 2011 IFIP, pp. 1-3. IEEE, 2011.

[2] Ko, Young-Bae, and Nitin H. Vaidya. "Location-Aided Routing (LAR) in mobile ad hoc networks." Wireless networks 6, no. 4 (2000): 307-321.

[3] Verma, Ravi Kumar, Ashish Xavier Das, and A. K. Jaiswal. "Effective Performance of Location Aided Routing Protocol on Random Walk (RW) Mobility Model using Constant Bit Rate (CBR)." International Journal of Computer Applications 122, no. 14 (2015).

[4] Hu, Lili, Zhizhong Ding, and Huijing Shi. "An improved GPSR routing strategy in VANET." In Wireless Communications, Networking and Mobile Computing (WiCOM), 2012 8th International Conference on, pp. 1-4. IEEE, 2012.

[5] Spaho, Evjola, Makoto Ikeda, Leonard Barolli, FatosXhafa, Muhammad Younas, and Makoto Takizawa. "Performance of OLSR and DSDV Protocols in a VANET Scenario: Evaluation Using CAVENET and NS3." In Broadband, Wireless Computing, Communication and Applications (BWCCA), 2012 Seventh International Conference on, pp. 108-113. IEEE, 2012.

[6] Toutouh, Jamal, and Enrique Alba. "Light commodity devices for building vehicular ad hoc networks: An experimental study." Ad Hoc Networks 37 (2016): 499-511.

[7] Wang, Yu, Jun Zheng, and Nathalie Mitton. "Delivery Delay Analysis for Roadside Unit Deployment in Vehicular Ad Hoc Networks with Intermittent Connectivity" (2016).

[8] Yang, Jianjun, and ZongmingFei. "Broadcasting with prediction and selective forwarding in vehicular networks." International journal of distributed sensor networks 2013 (2013). 